\title{
Inhibition of STAT5 induces G1 cell cycle arrest and reduces tumor cell invasion in human colorectal cancer cells
}

\author{
Hua Xiong ${ }^{1}$, Wen-Yu Su${ }^{1}$, Qin-Chuan Liang ${ }^{2}$, Zhi-Gang Zhang ${ }^{1}$, Hui-Min Chen ${ }^{1}$, Wan Du ${ }^{1}$, Ying-Xuan Chen ${ }^{1}$ and \\ Jing-Yuan Fang ${ }^{1}$
}

\begin{abstract}
Abnormalities in the signal transducer and activator of transcription (STAT) pathway are involved in the oncogenesis of several cancers. However, the mechanism by which dysregulated STAT5 signaling contributes to the progression of human colorectal cancer (CRC) has not been elucidated. To investigate the role of STAT5 in CRC progression, we depleted STAT5 with a small interfering RNA (siRNA). Our results demonstrate that STAT5 is involved in CRC cell growth, cell cycle

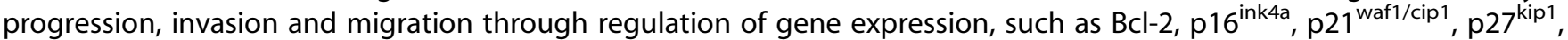
E-cadherin, the focal adhesion kinase (FAK), vascular endothelial growth factor (VEGF) and matrix metalloproteinases. In addition, immunohistochemical staining reveals upregulation of STAT5 during CRC tumorigenesis. Moreover, phosphoSTAT5 (pSTAT5) is predominantly localized in the cytoplasm of adenomas cells and colon adenocarcinoma cells, but primarily presented in the nucleus of normal colonic epithelium cells. Thus, pSTAT5 protein is shuttled from the nucleus to the cytoplasm in the oncogenesis of CRC, suggesting that activated STAT5 may also have cytoplasmic functions. In support of this hypothesis, we found that STAT5 formed a complex with p44/42 MAPK and SAPK/JNK in CRC cells, suggesting cross talk between STAT5 signaling and the MAPK pathway in the development of human CRC. Our findings illustrate the biological significance of STAT5 signaling in CRC progression, and provide novel evidence that intervention in STAT5 signaling may have potential therapeutic value in the prevention of human colorectal cancer.

Laboratory Investigation (2009) 89, 717-725; doi:10.1038/labinvest.2009.11; published online 16 March 2009
\end{abstract}

KEYWORDS: colorectal cancer; signal transduction; STAT5; tumorigenesis; tumor-related gene

Colorectal cancer (CRC) is a common malignancy and one of the leading causes of morbidity and death in the world. The development of more effective prevention and treatment strategies for CRC is limited by an incomplete understanding of the critical signaling pathways that are involved in CRC tumorigenesis. To date, despite our growing understanding of oncogenesis and successful identification of proto-oncogenes and tumor suppressor genes involved in the tumorigenesis of CRC, the biological and molecular mechanisms in CRC are still poorly understood. In general, the molecular mechanisms that control CRC progression are related to the altered expression of different proto-oncogenes, tumor suppressor genes, cytokines and their receptors, including Ras, Src, $\mathrm{p} 27^{\mathrm{kip} 1}, \mathrm{p} 16^{\mathrm{ink} 4 \mathrm{a}}$, interleukin (IL) and the epidermal growth factor receptor (EGFR). ${ }^{1-7}$ Notably, these abnormalities involve the signal transducers and activators of transcription (STAT) signaling pathway, specifically STAT3 and STAT5 signaling, although very few studies have reported the abnormal expression or activation of STAT proteins in colorectal cancer. ${ }^{8}$ Understanding the molecular mechanisms of STATs may further the development of novel therapies targeted in CRC treatment.

Signal transducers and activators of transcription is a family of transcription factors and is involved in a wide variety of cellular physiological processes, including differentiation, survival and cell growth., ${ }^{9,10}$ During the past few years, accumulating data have indicated constitutive activation of STAT 1,3 and 5 in a variety of diverse human tumor cell lines. In contrast to STAT1 function, considerable evidence suggests that constitutive activation of STAT3 and STAT5 is

\footnotetext{
'Department of Gastroenterology, Shanghai Jiao-Tong University School of Medicine Ren-Ji Hospital, Shanghai Institute of Digestive Disease, Shanghai, China and

${ }^{2}$ Department of Neurosurgery, Tangdu Hospital, Fourth Military Medical University, Xi'an, China

Correspondence: Professor J-Y Fang, Shanghai Institute of Digestive Disease, Ren-Ji Hospital, 145 Middle Shandong Road, Shanghai, 200001, China.

E-mail: jingyuanfang@yahoo.com

Received 16 July 2008; revised 6 December 2008; accepted 9 December 2008
} 
involved in tumor formation and progression. Like STAT3, STAT5 has been shown to regulate proliferation and inhibition of apoptosis in several cancer cell lines. ${ }^{11}$ For example, STAT5 activation has been shown primarily in hematopoietic malignancies, which are associated with the Bcl-Abl fusion protein. ${ }^{12}$ Subsequently, Li et al ${ }^{13}$ reported that STAT5 correlated with aggressiveness of prostate cancer These results indicate the significance of STAT5 signaling in tumorigenesis and progression. However, the mechanism by which dysregulated STAT5 signaling contributes to the progression of CRC has not been reported.

Given the significant role of STAT5 in carcinogenesis, we believe that abnormalities of STAT5 signaling may contribute to CRC oncogenesis. However, there have been no data regarding the role of STAT5 in CRC. In addition, downstream targets of STAT5 have still not been fully examined. This study was undertaken to determine the role of STAT5 in CRC tumorigenesis. To directly assess the biological significance of STAT5 signaling in CRC, we inhibited STAT5 with siRNA in two human CRC cell lines (SW1116 and HT29), and investigated changes in cell viability, apoptosis, cell cycle progression and cell invasive capability. We also evaluated changes in expression of several proteins that directly related to apoptosis (Bcl-2 and survivin), cell cycle regulation (p16 ${ }^{\text {ink4a }} \mathrm{p} 21^{\text {waf1/cip1 }}$ and $\mathrm{p} 27^{\mathrm{kip} 1}$ ) and cell invasion modulation (MMP2, MMP9, VEGF, FAK and E-cadherin). In addition, we also examined the expression of STAT5 and its active phosphorylated form in normal colonic epithelium, adenomas and primary colon adenocarcinomas. We sought to determine the role of STAT5 in CRC progression and test the hypothesis that STAT5 signaling may be targeted in colorectal cancer.

\section{MATERIALS AND METHODS}

\section{Cell Culture and Transient Transfection of STAT5 siRNA}

The two human colorectal cancer cell lines (SW1116 and HT29) used in this study were cultured in RPMI-1640 medium (Gibco, USA) or McCoy's 5A medium (Sigma, USA), respectively, supplemented with $10 \%$ fetal bovine serum and grown at $37^{\circ} \mathrm{C}$ in a humidified $5 \% \mathrm{CO}_{2}$ atmosphere.

To exclude an off-target effect and ensure the reproducibility of the results, two different commercial STAT5 small interfering RNAs were obtained from Dharmacon Inc. or Santa Cruz Biotechnology Inc. and used to target human STAT5 (accession numbers: NM_003152 STAT5a and NM_012448 STAT5b). Cells were transfected with siRNA $(50 \mathrm{nM})$ using the siPORT $^{\mathrm{TM}} \mathrm{NeoFX}^{\mathrm{TM}}$ transfection agent (Ambion) according to the manufacturer's instructions. Non-specific siRNA (Santa Cruz, CA, USA) was used as a negative control and the selective silencing of STAT5 was confirmed by western blot.

\section{Western Blot and Antibodies}

Whole-cell lysates were prepared from cancer cell lines, and standard western blot analysis was performed. ${ }^{14}$ Proteins were detected using the enhanced chemiluminescence detection kit (SuperSignal West Femto Substrate, Pierce). To ensure similar protein loading, the membrane was probed with a monoclonal antibody specific for GAPDH.

All antibodies in this study were purchased from Cell Signaling Technology Inc. (USA), except the following anti-

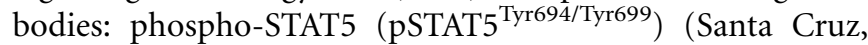
CA, USA), Bcl-2 (R\&D, USA) and GAPDH (Kangchen, China). All primary antibodies were used at a 1:1000 dilution.

\section{Cell Viability Assay}

Cell viability was assessed by a tetrazolium salt (WST-8)based colorimetric assay in the Cell Counting Kit 8 (CCK-8, Dojindo, Japan). ${ }^{15,16}$ Briefly, control and treated CRC cells were seeded onto 96-well plates at an initial density of $5 \times 10^{3}$ cells per well. At specified time points, $10 \mu \mathrm{l}$ of CCK-8 solution was added to each well of the plate, and the plate was incubated for $1 \mathrm{~h}$. Cell viability was determined by scanning with a microplate reader at $450 \mathrm{~nm}$. Data were expressed as the percentage of viable cells as follows: relative viability $(\%)=\left[\mathrm{A}_{450}(\right.$ treated $)-\mathrm{A}_{450} \quad($ blank $\left.)\right] /\left[\mathrm{A}_{450} \quad(\right.$ control $)-\mathrm{A}_{450}$ (blank) $] \times 100 \%$.

\section{Cell Cycle Analysis Using Propidium lodide and Flow Cytometry}

Approximately $1 \times 10^{6}$ cells were removed at specified time points, washed two times with PBS and fixed in cold ethanol for $30 \mathrm{~min}$ and then incubated with propidium iodide for 30 min prior to flow cytometer (BD, USA).

\section{Detection of Apoptosis}

Apoptosis was determined by flow cytometry analysis using annexin-V FITC and propidium iodide (PI) double staining assay in accordance with the manufacturer's instructions (Biovision, USA) as described earlier. ${ }^{14}$

\section{In Vitro Invasion Assay}

A cell invasion assay was performed as described earlier by Hecht et al. ${ }^{17}$ Briefly, cells were transfected with STAT5 siR$\mathrm{NA}(50 \mathrm{nM})$ for $48 \mathrm{~h}$, and then seeded $\left(1 \times 10^{5}\right.$ cells $)$ in $250 \mu \mathrm{l}$ of serum-free medium into the upper chamber of a transwell chamber. The lower chamber contained a medium supplemented with $10 \%$ fetal bovine serum as a chemo-attractant to induce invasion. Cells transfected with non-specific siRNA were used as the negative control. After incubation for $24 \mathrm{~h}$, non-migrated cells on the upper chamber of the filter were removed with a cotton swab, and migrated cells on the bottom surface of the filter were fixed, stained and counted.

\section{ELISA Analysis of VEGF, MMP2 and MMP9 Release}

Cells $\left(1 \times 10^{5}\right)$ transfected with STAT5 siRNA $(50 \mathrm{nM})$ were maintained in serum-free medium for $48 \mathrm{~h}$. The medium was collected, and the concentrations of vascular endothelial growth factor (VEGF), matrix metalloproteinases 2 (MMP2) and matrix metalloproteinases 9 (MMP9) in the medium 
were determined using an ELISA kit (R\&D systems, USA) according to the manufacturer's instruction.

\section{Tissue Microarray and Immunohistochemical Staining}

All specimens were collected from patients (38 primary colorectal adenocarcinomas and 46 adenomas) who underwent surgery in Shanghai Renji Hospital from July 2005 to January 2007. The protocol was approved by the ethics committee of Shanghai Jiao-Tong University School of Medicine, Renji Hospital and the research was carried out according to the provisions of the Helsinki Declaration of 1975. None of the patients received preoperative treatments, such as radiotherapy or chemotherapy. Fifteen specimens of normal colonic epithelium, taken from patients without colorectal cancer, were used as negative controls. Tissue microarray analysis (diameter, $1.0 \mathrm{~mm}$; depth, $4 \mu \mathrm{m}$ ) was performed by Outdo Biotech (Shanghai, China) using standard techniques. ${ }^{18}$

Tissue microarray sections were deparaffinized in xylene and rehydrated using a graded series of ethanol. A three-step streptavidin-biotin-horseradish peroxidase method was used, and the expressions of STAT5 and pSTAT5 ${ }^{\text {Tyr694/Tyr699 }}$ were examined with primary antibodies (STAT5, dilution 1:200; pSTAT5 $^{\text {Tyr694/Tyr699 }}$ dilution 1:100) using the LSAB + kit (DakoCytomation, Denmark) according to the manufacturer's instructions.

The slides were examined independently by two investigators blinded to both clinical and pathologic data. Protein expression was quantified using a visual grading system based on the extent of staining (percentage of positive tumor cells graded on a scale of $0-4$ : 0 , none; $1,1-25 \%$; $26-50 \% ; 3,51-75 \% ; 4,>75 \%)$ and the intensity of staining (graded on a scale of 0-3: 0, no staining; 1, weak staining; 2, moderate staining; 3 , strong staining). For further analysis, we used a product of grades of the extent and intensity of staining to define the cutoff value for high expression of the proteins, and the protein expression was classified into two categories: high (grades 4-12) and low (grades 0-3).

\section{Co-immunoprecipitation (Co-iP) Analysis}

To analyze the interaction between STAT5 and various cytoplasmic signaling effectors, such as p44/42 MAPK (ERK1 and ERK2), SAPK/JNK, p38 MAPK, PI3-kinase (PI3K) and AKT, the ProFound mammalian coimmunoprecipitation kit (Pierce, USA) was used in combination with standard western blotting techniques. Briefly, the supplied gel was coupled with $200 \mu \mathrm{g}$ of rabbit anti-human STAT5 antibody (Santa Cruz, CA, USA) or a non-relevant antibody (purified human $\mathrm{IgG}$, negative control). Cellular protein $(2.5 \mathrm{mg})$ from wholecell lysates was then added to the coupling gel for incubation. After the bound protein was eluted, the eluent was analyzed by western blot following incubation with the primary antibody and the appropriate HRP-conjugated secondary antibodies. Reciprocal immunoprecipitations were also performed as described above.

\section{Statistical Analysis}

Results are expressed as the mean \pm s.d. The data were analyzed for significance by ANOVA. Analysis comparing the expression of STAT5 or pSTAT5 ${ }^{\text {Tyr694/Tyr699 }}$ was performed with $\chi^{2}$ test and results were considered significant if the $\mu$-value was less than 0.05 . All analyses were performed with SPSS for Windows 13.0 software.

\section{RESULTS \\ RNAi Induces DownRegulation of STAT5 Signaling in CRC Cells}

We used two different siRNAs to selectively target STAT5. Similar reductions in STAT5 protein level were observed between the two siRNAs. Levels of both STAT5 and phosphorylated (activated) STAT5 decreased with time in both CRC cell lines. In SW1116 cells, for example, after being transfected with (Dharmacon) STAT5 siRNA, both STAT5 and pSTAT5 were depleted. The lowest levels of STAT5 expression were detected on day 3 , and the proteins were recovered at day 4 post-transfection (Figure 1a). We next examined the expressions of various apoptosis- and cell cycle-regulatory proteins known to be downstream targets of the STAT pathway. The overall pattern was similar for both siRNAs. As shown in Figure $1 \mathrm{~b}$, at $72 \mathrm{~h}$ post-transfection, the downregulation of STAT5 by siRNA (Santa Cruz) induced the downregulation of Bcl-2 that was simultaneously associated with the upregulation of $\mathrm{p} 16^{\text {ink4a }} \mathrm{p} 21^{\text {wafl/cip } 1}$ and p $27^{\text {kip1 }}$ in both CRC cells. However, no change in survivin was seen at the protein level.

\section{Inhibition of STAT5 Signaling by RNAi Suppresses CRC Cell Growth and Induces G1 Cell Cycle Arrest}

As detected by the CCK-8 assay (Figure 2a), RNAi-induced STAT5 deficiency inhibited CRC cell growth. This suppression lasted for $72 \mathrm{~h}$. The lowest percentage of cell viability was detected at $72 \mathrm{~h}$, although these cells recovered by $96 \mathrm{~h}$ posttransfection. These observations are consistent with STAT5 expression following siRNA treatment (Figure 1a), suggesting that STAT5 plays a significant role in CRC cell growth. To further explore the reason for the decrease in cell viability, we examined the effects of STAT5 on cell cycle progression and apoptosis. As illustrated in Figure 2b, pretreatment of CRC cells with STAT5 siRNA (Santa Cruz) blocked cell progression at the G1 phase of the cell cycle. In HT29 cells, for example, the G0/G1-phase fraction increased from 38.6\% (untreated) to $64.1 \%$ at $72 \mathrm{~h}$ post-transfection with $50 \mathrm{nM}$ of STAT5 siRNA, respectively. These observations are consistent with upregulation of $\mathrm{p} 16^{\mathrm{ink} 4 \mathrm{a}}, \mathrm{p} 21^{\mathrm{wafl} / \mathrm{cip} 1}$ and $\mathrm{p} 27^{\mathrm{kip} 1}$ expression, suggesting that the STAT5 pathway is involved in cell cycle regulation. We also performed flow cytometry analysis to evaluate whether the observed decrease in cell viability was due to apoptosis. However, there was no significant increase in apoptosis percentage in STAT5-transfected cells compared with the equivalent non-specific siRNA-transfected cells (data not shown). Therefore, our results indicate that the 


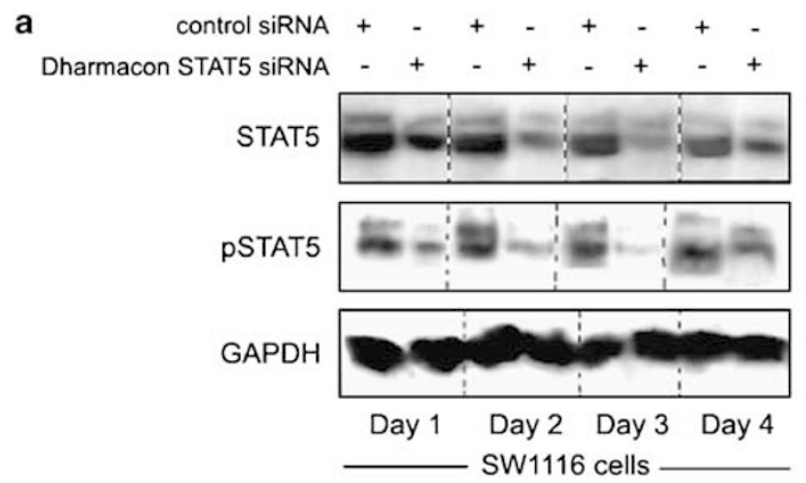

b

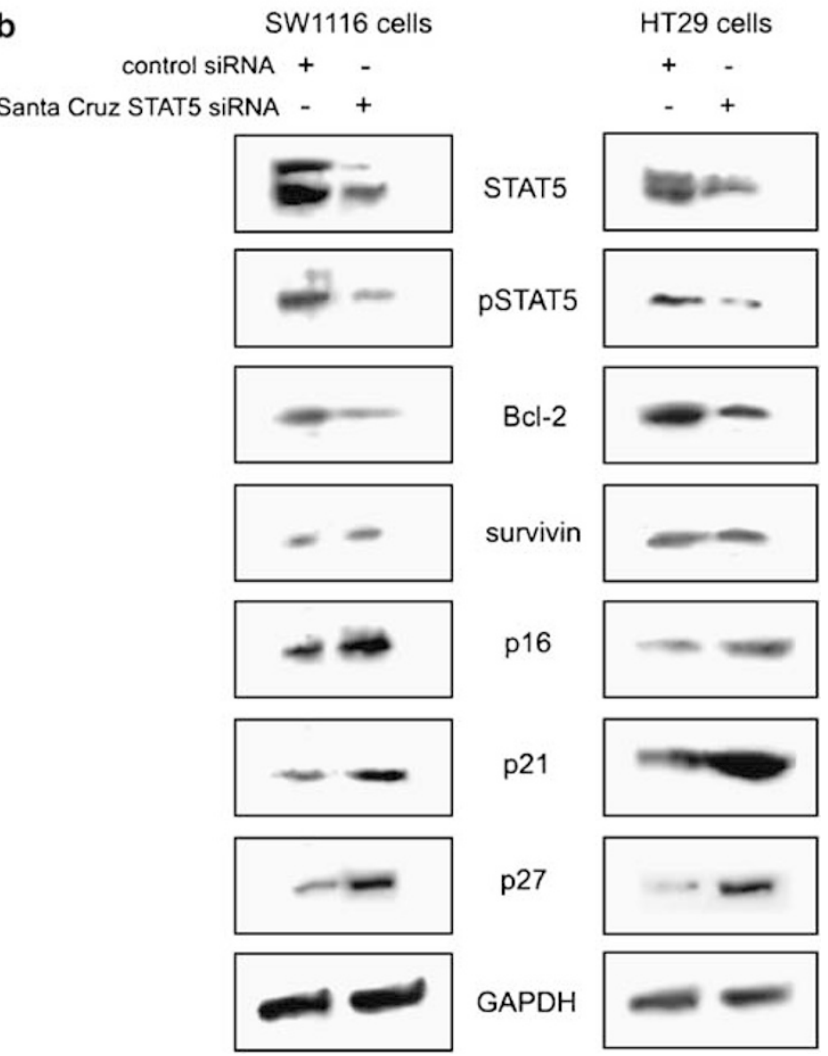

Figure 1 RNAi treatment decreases STAT5 signaling in CRC cells. (a) Western blot analysis demonstrated that both STAT5 and PSTAT5 were depleted in SW1116 cells after transfection with Dharmacon STAT5 siRNA. The lowest levels of STAT5 expression were detected on day 3 , and the proteins were recovered at day 4 post-transfection. (b) At $72 \mathrm{~h}$ posttransfection, western blot analysis showed that STAT5 siRNA (Santa Cruz) induced a downregulation of STAT5 and PSTAT5 in CRC cells. In addition, STAT5 siRNA-induced alterations in several, but not all downstream targets of STATs in CRC cells at $72 \mathrm{~h}$ after treatment. Bcl-2 protein was downregulated simultaneous to an upregulation of p16 $6^{\text {ink4a }}, \mathrm{p} 21^{\text {waf1/cip } 1}$ and $p 27^{k i p 1}$. Survivin protein showed no detectable change. The data shown are representative of three separate experiments. GAPDH was used for the loading control in all experiments.

STAT5-induced decrease in cell viability can be attributable at least in part to the occurrence of G1 cell cycle arrest after inhibition of STAT5 expression.

\section{Disruption of STAT5 Signaling by RNAi Inhibits Colorectal Cancer Cells Invasion}

We next addressed the functional role of STAT5 in the invasion of cells into the surrounding tissue. As shown in Figure 3a, treatment of cells with STAT5 siRNA (Santa Cruz or Dharmacon), but not non-specific siRNA, effectively suppressed CRC cell invasion. For instance, $48 \mathrm{~h}$ after transfection with $50 \mathrm{nM}$ of STAT5 siRNA (Santa Cruz), the number of SW1116 and HT29 cells able to migrate through the filter decreased to 63.5 and $57.4 \%$, respectively, when compared to cells transfected with non-specific siRNA. These results indicate that STAT5 is a critical mediator involved in JAK/STAT-induced cell invasion.

\section{Decreased STAT5 Activation is Associated with Modulation of E-Cadherin, FAK, VEGF and MMP2, but not MMP9}

To further understand the mechanisms of STAT5 signaling in CRC cell invasion and reveal downstream events of STAT5 signaling involved in the regulation of cell invasion, we examined the expression of various migration and invasion regulatory proteins by western blot and ELISA. As shown in Figure 3b and c, STAT5 siRNA (Santa Cruz) induced a loss of expression of FAK, pFAK ${ }^{\text {Tyr397 }}$ VEGF and MMP2. However, the same treatment led to an increase in E-cadherin expression in CRC cells (Figure $3 \mathrm{~b}$ and c). However, no significant change in MMP9 secretion was seen in cells treated with STAT5 RNAi (Figure 3c). Similar patterns were seen following Dharmacon siRNA transfection.

\section{Adenomas and Colon Adenocarcinomas showed Higher Expression of STAT5 Compared to Normal Colonic Mucosa}

Table 1 shows the expression frequency of STAT5 and pSTAT5 by immunohistochemical staining. STAT5 expression was detected mainly in the cytoplasm (Figure 4). High levels of STAT5 were detected in $66.7 \%$ of the normal colonic epithelium samples, in $84.8 \%$ of the adenoma samples and in $94.7 \%$ of the primary colon adenocarcinoma samples. Thus, adenomas and colon adenocarcinomas showed higher expression of STAT5 than normal colonic mucosa $(P<0.05)$, suggesting that upregulation of STAT5 in colon adenocarcinoma may have important implications in colorectal cancer biology.

\section{Activated STAT5 (pSTAT5) is Localized to the Cytoplasm of Adenoma Cells and Colon Adenocarcinomas Cells, Although Predominantly a Nuclear Protein in Normal Epithelial Cells}

We tested two different anti-human pSTAT5 antibodies from different manufacturers with the similar results. Although there was no significant difference in the level of pSTAT5 between these samples, pSTAT5 showed a change in localization. In $91.3 \%$ of the adenoma samples and in $100 \%$ of the primary colon adenocarcinoma, pSTAT5 was predominantly 

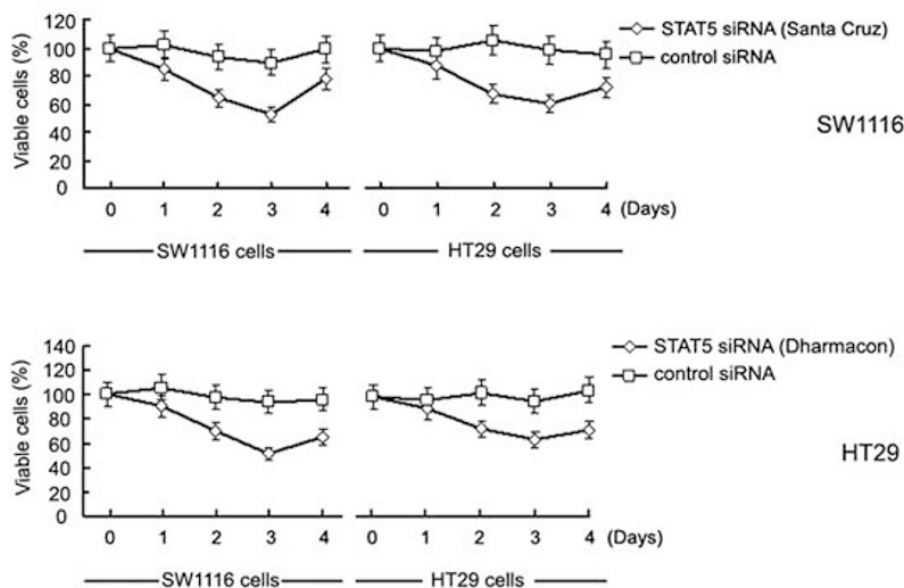

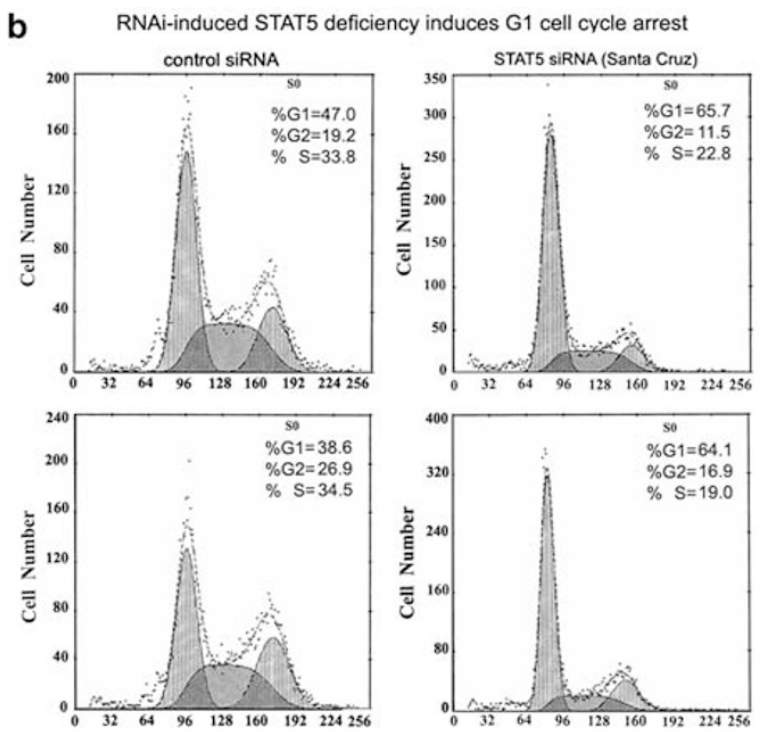

Figure 2 The functional role of STAT5 signaling on CRC cell growth and cell cycle progression. (a) Cell viability was determined by the CCK-8 assay following treatment of CRC cells with STAT5 siRNAs. The percentage of viable cells was determined as described in the Materials and Methods section. STAT5 siRNA inhibited CRC cell growth. This suppression persisted for $72 \mathrm{~h}$, after which the cell recovered at $96 \mathrm{~h}$ post-transfection. The results represent mean \pm s.d. of three experiments. (b) Cell cycle analysis was performed after treating CRC cells with STAT5 siRNA (Santa Cruz). STAT5 knockdown led to a block in the cell cycle at the $\mathrm{G} 1$ phase at $72 \mathrm{~h}$ after transfection.

cytoplasmic, whereas the same protein was found to be predominantly nuclear in $86.7 \%$ of the normal colonic epithelium samples (Figure 4). Thus, our results show a significant correlation between pSTAT5 localization and tissue type, suggesting that the activated STAT5 protein is shuttled from the nucleus to the cytoplasm in the oncogenesis of CRC. These observations suggest that oncogenic STAT5 activity may not only involve a nuclear function (as a transcription factor), but cytoplasmic functions as well (eg, cross talk with members of other pathway).

\section{Interaction of STAT5 with p44/42 MAPK and SAPK/JNK in CRC Cells}

Before exploring the cytoplasmic function of STAT5 in the oncogenesis of CRC, we hypothesized that STAT5 proteins may contribute to CRC through interactions with various signaling molecules. To verify this hypothesis, we performed co-immunoprecipitation studies and found that STAT5 formed a complex with p44/42 MAPK and SAPK/JNK in SW1116 human colorectal cancer cells (Figure 5). Thus, our data indicate that STAT5 interacts with ERK1/ERK2 and SAPK/JNK in CRC cells. Furthermore, the results also imply a cross talk between STAT5 signaling and the MAPK pathway in CRC. In addition, although STAT5 was present in the PI3K immunoprecipitates in leukemic bone marrow cells, ${ }^{19}$ the STAT5-PI3K complex was not observed in this system. Of note, the STAT5-p38 complex and STAT5-AKT complex were not detected in our studies (Figure 5).

\section{DISCUSSION}

Despite improvements in determining the molecular mechanisms of CRC tumorigenesis, the specific signal transduction pathways involved in CRC have not been fully characterized. ${ }^{20}$ Recently, constitutive activation of STAT proteins has been observed in several malignant neoplasms. $^{21-23}$ Like STAT3, STAT5 has been shown to actively participate in tumor development and progression. ${ }^{24-27}$ However, the precise role of STAT5 signaling in human CRC progression has not been characterized. In this study, we provide experimental and mechanistic evidence that abnormalities of STAT5 signaling contribute to the initiation and progression of CRC.

In this study, we first evaluated the biological significance of STAT5 signaling in the pathogenesis of CRC cells. Although both originated from colorectal adenocarcinoma, the SW1116 and HT29 cell lines are not isogenic (that is, HT29 cell is positive for the expression of $\mathrm{N}$-ras oncogene, whereas $\mathrm{N}$-ras is not detected in SW1116 cells; in HT29 cells, there is a $\mathrm{G}$ to A mutation in codon 273 of the p53 gene resulting in an Arg to His substitution, however, SW1116 contains WT p53), and these two cell lines have different genetic and epigenetic backgrounds. Therefore, two different CRC cell lines were chosen in our studies. To exclude an off-target effect and ensure the reproducibility of the results, two different commercial STAT5 siRNAs were used to selectively block STAT5 signaling. Our results indicate that downregulation of STAT5 and pSTAT5 was associated with a gradual decrease in cell viability. Moreover, the decrease in cell viability can be attributed to the occurrence of G1 cell cycle arrest following 

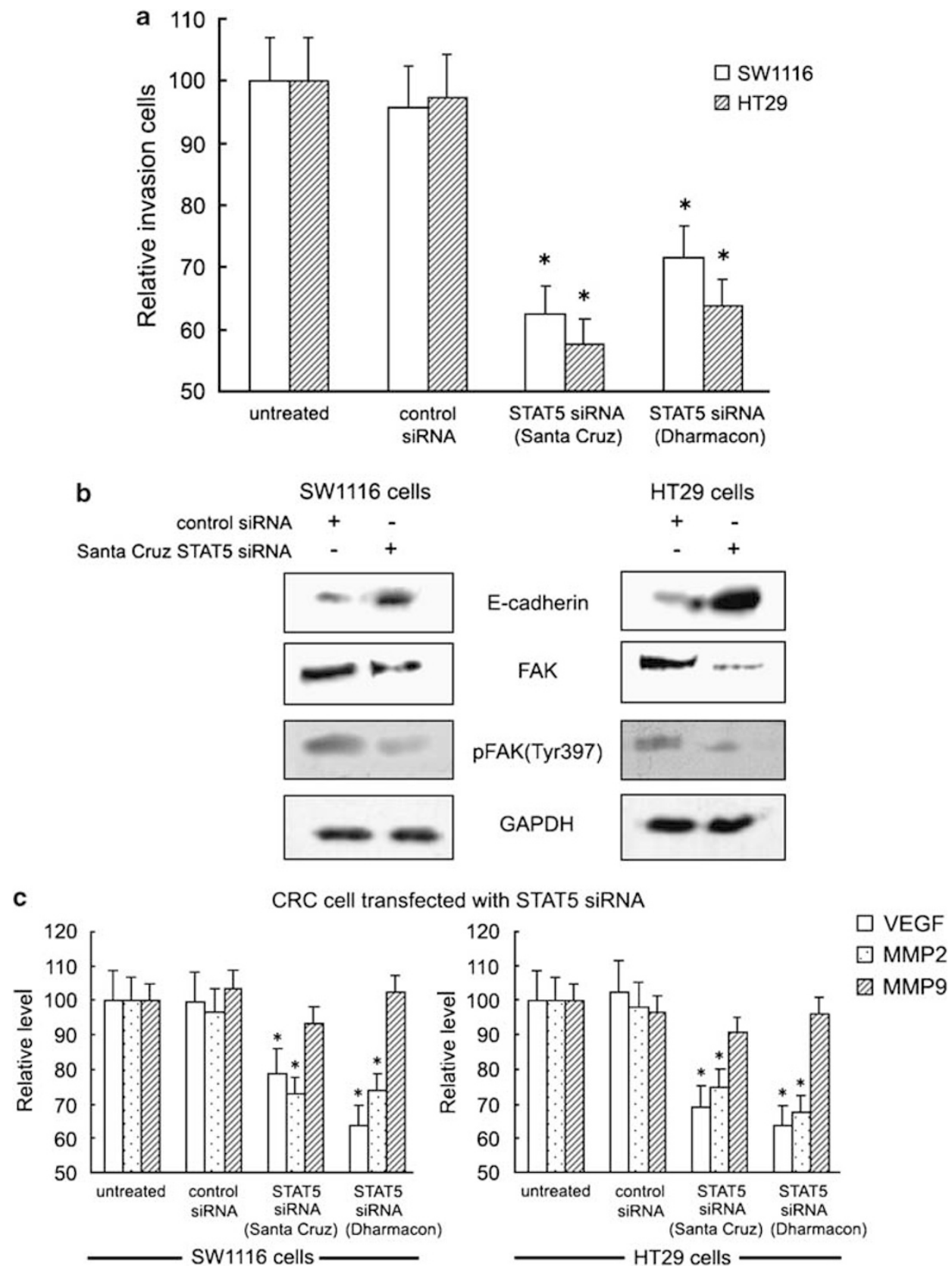

Figure 3 The functional role of STAT5 signaling on the invasive ability of CRC cells. (a) CRC cells transfected with STAT5 siRNA were examined for their invasive capability. At $48 \mathrm{~h}$ post-transfection, the number of migrated cells significantly decreased compared with untreated cells $\left({ }^{*} P<0.05\right)$. (b) Western blot analysis showed that STAT5 siRNA (Santa Cruz) induced an upregulation of E-cadherin, simultaneous with a downregulation of FAK in CRC cells at $72 \mathrm{~h}$ post-transfection of the cells. (c) Effects of STAT5 siRNA on the secretion of VEGF, MMP2 and MMP9. At 48 $\mathrm{h}$ post-transfection, the concentrations of VEGF and MMP2 were decreased compared with that of untreated cells $\left({ }^{*} P<0.05\right)$. The experiment was performed three times with consistent findings.

the inhibition of STAT5 signaling. These data are consistent with the upregulation of $\mathrm{p} 16^{\mathrm{ink} 4 \mathrm{a}}, \mathrm{p} 21^{\text {wafl/cip1 }}$ and $\mathrm{p} 27^{\mathrm{kip} 1}$ expression following suppression of STAT5, suggesting that the STAT5 pathway is involved in cell cycle regulation. In addition, despite the decrease in Bcl-2, we did not detect a significant apoptosis in STAT5 depleted cells. This result can be, at least partially, explained by the persistent expression of survivin. Our data indicate that STAT5 is essential, but not sufficient, for the survival of CRC cells.

Invasiveness is a key step that leads to metastasis, resulting in poor prognosis. ${ }^{28}$ Thus, the study of the molecular mechanism of CRC invasiveness is of great value. Results from the Matrigel invasion assay showed an inverse relationship between the invasiveness of CRC cell and inhibition of STAT5, demonstrating 
a potential regulatory effect of STAT5 signaling on the invasive capability of CRC cells. Furthermore, our data suggest that blockade of STAT5 activity decreases the expression of FAK, MMP2 and VEGF, but increases the expression of E-cadherin, suggesting that STAT5 may be involved in the regulation of the expressions of FAK, MMP2, VEGF and E-cadherin. These results imply that STAT5 signaling may regulate multiple processes in CRC invasion. First, stimulating MMP-2 production could induce degradation of the extracellular matrix. Second, STAT5 could regulate CRC invasive capability by affecting angiogenesis through VEGF. Third, STAT5 may also function in tumor metastasis and invasion by regulating E-cadherin, a protein belonging to the family of cell-cell adhesion molecules involved in the maintenance of cell differentiation. Thus, we provide mechanistic evidence that STAT5 signaling may affect CRC metastasis by multiple mechanisms including enzyme-

Table 1 Expression of STAT5 and pSTAT5

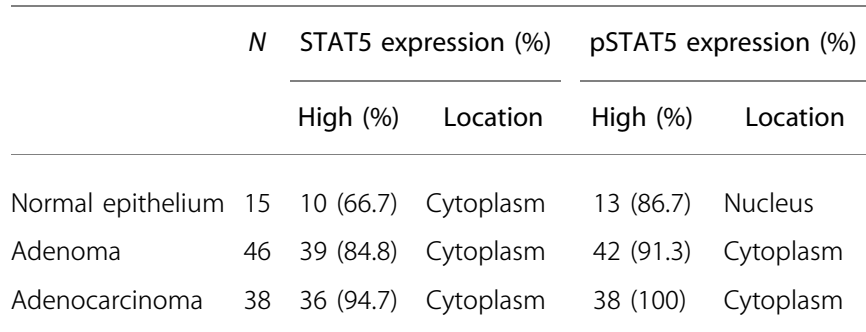

STAT5 and PSTAT5 expressions levels were frequently detected in normal colonic epithelium, adenoma and primary colon adenocarcinoma. STAT5 staining was detected primarily in the cytoplasm, with occasional nuclear staining. PSTAT5 predominantly localized to the cytoplasm of adenoma cells and colon adenocarcinoma cells, but mostly presented in the nucleus of normal colonic epithelium cells. based degradation of the extracellular matrix, angiogenesis, adhesion and migration.

For the first time, immunohistochemical staining revealed an upregulation of STAT5 during CRC tumorigenesis. However, our data did not confirm the results performed in human prostate cancer cells reported by Ahonen $e a^{29}$ who reported that the level of pSTAT5 increased in prostate cancer cells compared with adjacent normal prostate epithelial cells. These authors found no localization change and that pSTAT5 was generally present in the nucleus of both prostate cancer cells and normal prostate epithelial cells. Nevertheless, our data revealed a significant correlation between pSTAT5 localization and tissue type, as activated STAT5 localizes to the cytoplasm of adenoma cells and colon adenocarcinoma cells, whereas it is found in the nucleus of normal epithelium cells. However, no significant difference in the level of pSTAT5 was observed between normal epithelium cells and adenoma cells and colon adenocarcinoma cells. These conflicting data imply that STAT5 plays a complex role in CRC tumorigenesis. Based on the pSTAT5 protein expression in CRC tissues, we hypothesized that oncogenic STAT5 activity may involve not only a nuclear function (as a transcription factor), but also a cytoplasmic function (eg, cross talk with members of other pathway). Accordingly, we also found that STAT5 forms a complex with ERK1/ERK2 and SAPK/JNK in CRC cells, implying cross talk between STAT5 signaling and the MAPK pathway in the development of human CRC. However, a recent report documented that STAT5 was present in the PI3K immunoprecipitates in leukemic bone marrow cells, ${ }^{19}$ but the STAT5-PI3K complex was not observed in this system. This may be the reason that the STAT5 activity depends on the specific cell type and tumor microenvironment.
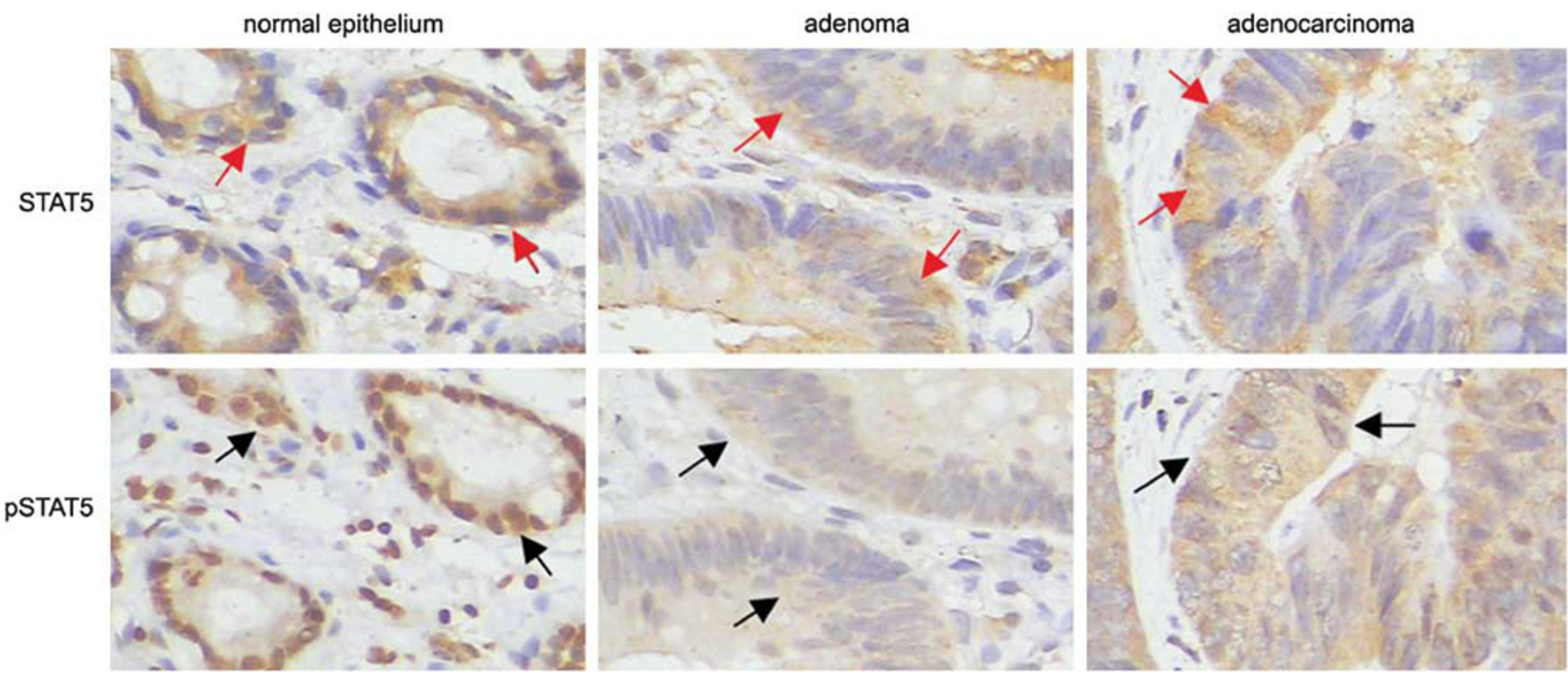

Figure 4 Immunohistochemical staining of the tissue microarray. Predominantly cytoplasmic staining of STAT5 was detected in normal colonic epithelium, adenomas and primary colon adenocarcinomas (as indicated by red arrows). pSTAT5 localized to the cytoplasm of adenoma cells and colon adenocarcinoma cells, although predominantly found in the nucleus of normal epithelium cells (Black arrows indicate pSTAT5 protein is shuttled from nucleus to cytoplasm in the oncogenesis of CRC; magnification $=\times 400$ ). 
a

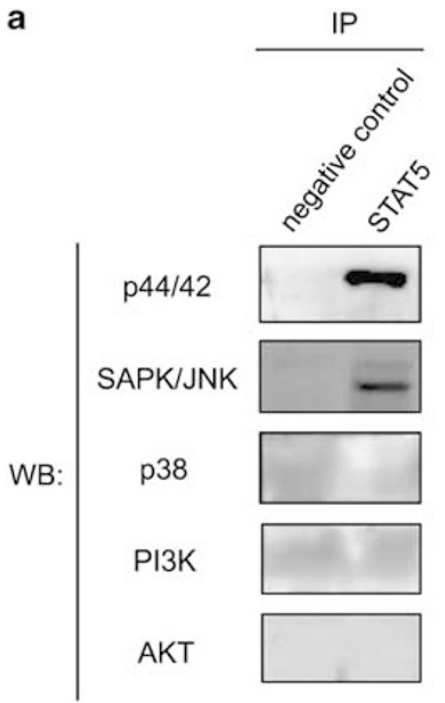

b

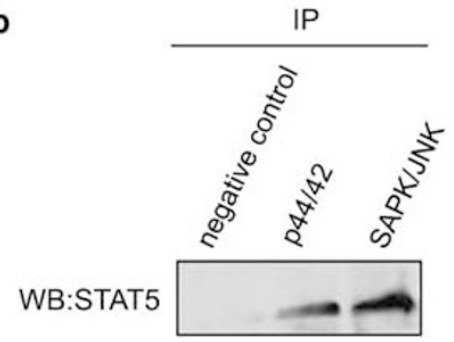

Figure 5 Interaction of STAT5 with MAPK, PI3K and AKT in CRC cells. (a) Protein-protein interactions were determined by co-immunoprecipitation analysis. Co-immunoprecipitation analysis revealed that STAT5 formed a complex with p44/42 MAPK and SAPK/JNK in SW1116 cells. However, no significant interactions of STAT5 with PI3K, p38, AKT were detected in our studies. (b) Reciprocal immunoprecipitations were performed with antibodies directed against $\mathrm{p} 44 / 42$ or SAPK/JNK or a non-relevant antibody (purified human IgG, negative control), then detected STAT5 by western blot. These results also validate the interactions of STAT5 with p44/42 MAPK or SAPK/JNK in CRC cells.

In conclusion, this study is the first to examine the mechanistic role of STAT5 in CRC tumorigenesis and progression. Our work suggests that blocking STAT5 activity in human CRC is a novel therapeutic approach, as STAT5 is implicated in many areas of tumor progression including cell growth, cell cycle regulation, invasion and migration. Moreover, the finding that STAT5 may cross talk with members of other pathways, such as MAPK, indicates that STAT5 is embedded in complex signaling networks. Thus, our present findings strongly suggest that STAT5 signaling plays a significant role in CRC malignant progression, and intervention in STAT5 signaling may have potential therapeutic value in the prevention of human colorectal cancer.

\section{ACKNOWLEDGEMENTS}

This study was supported by grants from the National Basic Research Program of China (973 program) (no: 2005CB522400), the National Science Fund for Distinguished Young Scholars to FJY (no: 30625034), and the National Natural Science Foundation of China (no: 30800513). Our special thanks to Ms Hongyin Zhu for excellent technical assistance and enthusiastic participation in this study.

1. Maurer GD, Leupold JH, Schewe DM, et al. Analysis of specific transcriptional regulators as early predictors of independent prognostic relevance in resected colorectal cancer. Clin Cancer Res 2007;13:1123-1132.

2. Caron RW, Yacoub A, Li M, et al. Activated forms of H-RAS and K-RAS differentially regulate membrane association of PI3K, PDK-1, and AKT and the effect of therapeutic kinase inhibitors on cell survival. Mol Cancer Ther 2005;4:257-270.

3. Lyall MS, Dundas SR, Curran S, et al. Profiling markers of prognosis in colorectal cancer. Clin Cancer Res 2006;12:1184-1191.

4. Kaspar M, Trachsel E, Neri D. The antibody-mediated targeted delivery of interleukin-15 and GM-CSF to the tumor neovasculature inhibits tumor growth and metastasis. Cancer Res 2007;67: 4940-4948.

5. Schmelz EM, Xu H, Sengupta $R$, et al. Regression of early and intermediate stages of colon cancer by targeting multiple members of the EGFR family with EGFR-related protein. Cancer Res 2007;67: 5389-5396.

6. Galizia G, Lieto E, De Vita F, et al. Cetuximab, a chimeric human mouse anti-epidermal growth factor receptor monoclonal antibody, in the treatment of human colorectal cancer. Oncogene 2007;26:3654-3660.

7. Dambacher J, Beigel F, Seiderer J, et al. Interleukin-31 mediates MAP kinase and STAT1/3 activation in intestinal epithelial cells and its expression is upregulated in inflammatory bowel disease. Gut 2007;56:1257-1265.

8. Spano JP, Milano G, Rixe C, et al. JAK/STAT signalling pathway in colorectal cancer: a new biological target with therapeutic implications. Eur J Cancer 2006;42:2668-2670.

9. Clevenger CV. Roles and regulation of stat family transcription factors in human breast cancer. Am J Pathol 2004;165:1449-1460.

10. Lee TK, Man K, Poon RT, et al. Signal transducers and activators of transcription $5 \mathrm{~b}$ activation enhances hepatocellular carcinoma aggressiveness through induction of epithelial-mesenchymal transition. Cancer Res 2006;66:9948-9956.

11. Xi S, Zhang $Q$, Gooding $W E$, et al. Constitutive activation of Stat5b contributes to carcinogenesis in vivo. Cancer Res 2003;63:6763-6771.

12. Coffer PJ, Koenderman L, de Groot RP. The role of STATs in myeloid differentiation and leukemia. Oncogene 2000;19:2511-2522.

13. Li $\mathrm{H}$, Zhang $\mathrm{Y}$, Glass $\mathrm{A}$, et al. Activation of signal transducer and activator of transcription-5 in prostate cancer predicts early recurrence. Clin Cancer Res 2005;11:5863-5868.

14. Lu R, Wang X, Chen ZF, et al. Inhibition of the extracellular signalregulated kinase/mitogen-activated protein kinase pathway decreases DNA methylation in colon cancer cells. J Biol Chem 2007;282: 12249-12259.

15. Morita $Y$, Naka T, Kawazoe $Y$, et al. Signals transducers and activators of transcription (STAT)-induced STAT inhibitor-1 (SSI-1)/suppressor of cytokine signaling-1 (SOCS-1) suppresses tumor necrosis factor alpha-induced cell death in fibroblasts. Proc Natl Acad Sci USA 2000;97:5405-5410.

16. Wang YY, Zhou GB, Yin T, et al. AML1-ETO and C-KIT mutation/ overexpression in $t(8 ; 21)$ leukemia: implication in stepwise leukemogenesis and response to Gleevec. Proc Natl Acad Sci USA 2005;102:1104-1109.

17. Hecht M, Papoutsi M, Tran HD, et al. Hepatocyte growth factor/c-Met signaling promotes the progression of experimental human neuroblastomas. Cancer Res 2004;64:6109-6118.

18. Yamabuki T, Takano A, Hayama S, et al. Dikkopf-1 as a novel serologic and prognostic biomarker for lung and esophageal carcinomas. Cancer Res 2007;67:2517-2525.

19. Harir N, Pecquet C, Kerenyi M, et al. Constitutive activation of Stat5 promotes its cytoplasmic localization and association with PI3-kinase in myeloid leukemias. Blood 2007;109:1678-1686.

20. Oving IM, Clevers HC. Molecular causes of colon cancer. Eur J Clin Invest 2002;32:448-457.

21. Saxena NK, Sharma D, Ding X, et al. Concomitant activation of the JAK/ STAT, PI3K/AKT, and ERK signaling is involved in leptin-mediated promotion of invasion and migration of hepatocellular carcinoma cells. Cancer Res 2007;67:2497-2507. 
22. Pecquet $C$, Nyga $R$, Penard-Lacronique $V$, et al. The Src tyrosine kinase $\mathrm{Hck}$ is required for Tel-Abl- but not for Tel-Jak2-induced cell transformation. Oncogene 2007:26:1577-1585.

23. Walker SR, Nelson EA, Frank DA. STAT5 represses BCL6 expression by binding to a regulatory region frequently mutated in lymphomas. Oncogene 2007;26:224-233.

24. Bromberg J, Darnell Jr JE. The role of STATs in transcriptional control and their impact on cellular function. Oncogene 2000;19: 2468-2473.

25. Turkson J, Jove R. STAT proteins: novel molecular targets for cancer drug discovery. Oncogene 2000;19:6613-6626.
26. Catlett-Falcone R, Dalton WS, Jove R. STAT proteins as novel targets for cancer therapy. Signal transducer an activator of transcription. Curr Opin Oncol 1999;11:490-496.

27. Bowman T, Garcia R, Turkson J, et al. STATs in oncogenesis. Oncogene 2000;19:2474-2488.

28. Tung-Ping Poon R, Fan ST, Wong J. Risk factors, prevention, and management of postoperative recurrence after resection of hepatocellular carcinoma. Ann Surg 2000;232:10-24.

29. Ahonen TJ, Xie JW, LeBaron MJ, et al. Inhibition of transcription factor Stat5 induces cell death of human prostate cancer cells. J Biol Chem 2003;278:27287-27292. 